\title{
Self-Stabilizing Silicon Nitride Lightsails
}

\author{
Ramon Gao ${ }^{1}$, Yonghwi Kim², Laura Kim ${ }^{1,3}$, Michael D. Kelzenberg ${ }^{1}$, Ognjen Ilic ${ }^{1,4}$, Harry A. Atwater ${ }^{1}$ \\ ${ }^{1}$ Department of Applied Physics and Materials Science, California Institute of Technology, Pasadena, CA 91125, USA \\ ${ }^{2}$ Department of Electrical Engineering, California Institute of Technology, Pasadena, CA 91125, USA \\ ${ }^{3}$ Department of Electrical Engineering and Computer Science, Massachusetts Institute of Technology, Cambridge, MA 02139, USA \\ ${ }^{4}$ Department of Mechanical Engineering, University of Minnesota, Minneapolis, MN 55455, USA \\ rggao@caltech.edu,haa@caltech.edu
}

\begin{abstract}
We report a design for a microscopic lightsail prototype that allows for passive stabilization in the radiation-pressure dominated regime. Stable dynamics of our silicon nitride structure are predicted for initial tilts of up to $\pm 10^{\circ}$. $\odot 2020$ The Author(s)
\end{abstract}

\section{Introduction}

Successful propulsion of macroscopic ultrathin laser-driven lightsails to high speeds, as envisioned by the Breakthrough Starshot initiative, will critically depend on the ability to passively stabilize their dynamics during propulsion [1]. Recently, stable acceleration of ultralight objects via laser radiation pressure has been reported by employing Bloch-wave type scatterers [2], beam-steering optical metasurfaces [3], and diffractive lightsails [4]. In addition to the stabilization mechanism, the exerted pressure can be enhanced by structuring the sail surface with asymmetric resonant cavity arrays [5]. As for realizing microscopic lab-scale lightsail prototypes, the choice of a suitable material and the photonic design for passive stability depict two key challenges.

Among the potential material candidates, for example silicon or $\mathrm{MoS}_{2}$, silicon nitride exhibits desirable optical properties in the visible wavelength regime. While low absorption losses are crucial for the thermal management of the lightsail, a sufficiently large index contrast will result in an efficient photon momentum transfer during propulsion. With an extinction coefficient of below $10^{-3}$ and a refractive index of 2.05 at $514 \mathrm{~nm}$, silicon nitride meets these requirements. Moreover, silicon nitride membranes have been employed as high- $Q$ mechanical resonators in optomechanical experiments [6], and are known to be mechanically robust and suitable for nanofabrication.

In this work, we report a tailored design that comprises silicon nitride resonators on a silicon nitride substrate with an engineered optical anisotropy of scattering to obtain passive stabilization in the radiation-pressure dominated regime. Using the finite-element-method software COMSOL Multiphysics, our numerical electromagnetic simulations explore the influence of the light-induced forces and torque of our design on the lightsail trajectory for a range of initial lateral displacements and angular tilts with respect to the center of an unstructured beam.

\section{Discussion}

Our design is based on an asymmetric unit cell that consists of two optical, subwavelength-thick silicon nitride resonators on a $100 \mathrm{~nm}$ thick silicon nitride substrate (Fig. 1). By arranging these unit cells to be mirror-symmetric with respect to the structure's center-of-mass, we engineered its optical anisotropy of scattering to obtain selfrestoring forces and torque. Using COMSOL, we calculated the light-induced optical pressures in lateral and vertical directions $p_{x, z}$ via integration of the Maxwell Stress tensor over the surface of a box enclosing our structure, from which the lateral and vertical forces $F_{x, z}$ as well as the torque $\tau_{y}$ can be derived.

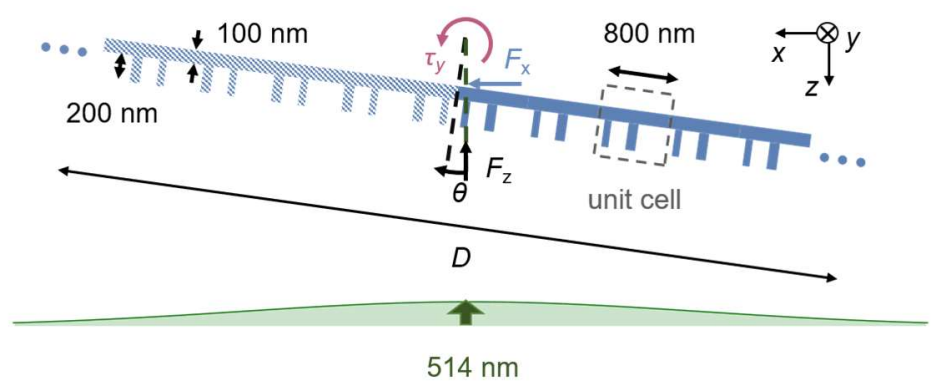

Figure 1: Schematic of the silicon nitride lightsail design. 
By systematically varying the geometrical parameters of the resonators, we identified a design whose lateral force and torque profiles hint at a self-restoring mechanism upon initial angular tilts with respect to the laser beam center of up to $\pm 10^{\circ}$ (Fig. 2a). To confirm this set of stable initial conditions, we numerically evolved the equations of motion based on the knowledge of $F_{x, z}(\theta, x)$ and $\tau_{y}(\theta, x)$, where $x$ denotes the lateral displacement of the structure from the beam center. Bounded dynamics can be observed by plotting the trajectory of our lightsail for a given initial tilt $\theta_{0}$ and lateral displacement $x_{0}$ versus time. For example, an initial tilt of $-7.3^{\circ}$ and lateral displacement of $-0.12 D$, where $D$ is the diameter of our structure, will still result in stable dynamics due to the restoring force and torque that arise from anisotropically scattered light (Fig. 2b). More extreme angular tilts such as $-10^{\circ}$ can be compensated for by initially displacing the lightsail accordingly, i.e. by at least $0.4 D$ and at most $1.3 D$. By permuting possible combinations of initial conditions $\theta_{0}$ and $x_{0}$, we identified a continuous and asymmetric domain of stable initial conditions for our design.

To characterize the dynamics of our silicon nitride design and probe the light-induced forces and torque, we will fabricate resonators on suspended membranes. We studied the expected displacement of the membrane suspended on optimized serpentine springs for enhanced compliance at various incident power densities in COMSOL. For example, at a power density of close to $5 \mathrm{~kW} / \mathrm{cm}^{2}$, we predict the center of the membrane to be deflected by $140 \mathrm{~nm}$. Such displacements will be accompanied by a rise of membrane temperature, which we simulated to be below the melting point of silicon nitride at relevant incident power densities.

First suspended structures without the resonators comprising a square $40 \mu \mathrm{m} \mathrm{x} 40 \mu \mathrm{m}$ center membrane attached to four serpentine springs have been successfully fabricated (Fig. 2c). To verify our proposed theoretical design for dynamical stability, we will present first experimental results for the optical spectrum using spectral and angular resolved photocurrent measurements.

(a)

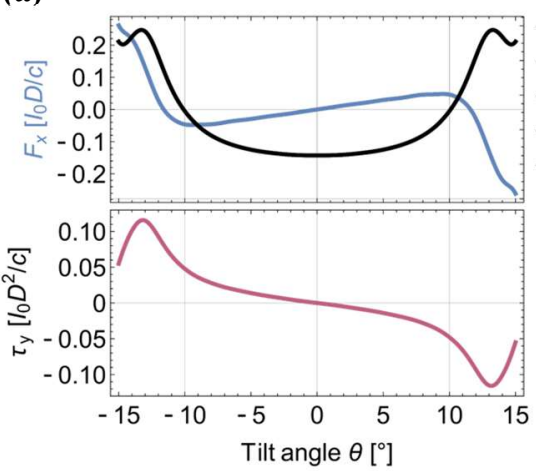

(b)
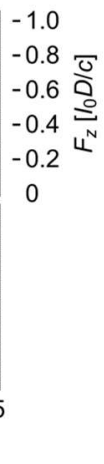

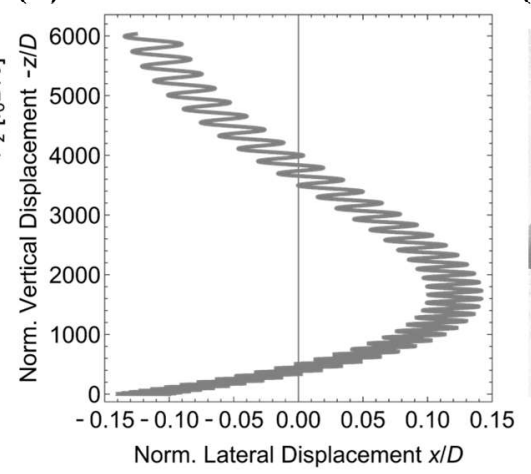

(c)

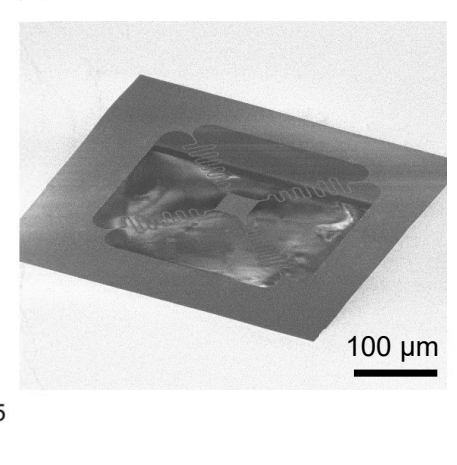

Figure 2: a) Lateral and vertical light-induced forces and torque versus tilt angle. b) Bounded trajectory for a lightsail displaced laterally by $-0.12 D$ and tilted by $-7.3^{\circ}$. c) SEM image of a suspended plain Silicon Nitride membrane attached to serpentine springs.

\section{Conclusion}

In summary, we have studied the feasibility of passively stabilizing an all-silicon nitride lightsail by tailoring the design of the resonators within an asymmetric unit cell for self-restoring light-induced forces and torque. Our calculated trajectories suggest that self-stabilization can be achieved for a range of initial conditions, i.e. tilts of up to $\pm 10^{\circ}$. These results serve as guidelines to the fabrication of first prototype lightsails, whose dynamical stability we plan to assess within our suspended silicon nitride membrane platform.

\section{References}

[1] H. A. Atwater et al., Nature Materials 17, 861-867 (2018)

[2] O. Ilic, H. A. Atwater, Nature Photonics 13, 289-295 (2019)

[3] J. Siegel et al., ACS Photonics 6, 2032-2040 (2019)

[4] P. R. Srivastava, et al., Optics Letters 44.12, 3082-3085 (2019)

[5] L. F. Yang, et al., Physical Review Letters 122, 083901 (2019)

[6] R. A. Norte et al., Physical Review Letters 116, 147202 (2016) 